\title{
A new colocated finite volume scheme for the incompressible Navier-Stokes equations on general non matching grids
}

\author{
Robert Eymard ${ }^{\mathrm{a}}$, Raphaèle Herbin ${ }^{\mathrm{b}}$ \\ aniversité de Marne-la-Vallée, 77454 Marne-la-Vallée cedex 2, France \\ b Université de Provence, 39 rue Joliot-Curie, 13453 Marseille, France \\ Received *****; accepted after revision +++++ \\ Presented by Philippe G. Ciarlet
}

\begin{abstract}
A new finite volume scheme is used for the approximation of the Navier-Stokes equations on general grids, including non matching grids. It is based on a discrete approximation of the weak form and on the definition of discrete gradient and divergence operators on each control volume. A sketch of the convergence proof is given, and the results of a numerical implementation on a non matching grids are shown. A byproduct is a finite volume scheme that is convergent for diffusion problems on general grids. To cite this article: R. Eymard, R. Herbin, C. R. Acad. Sci. Paris, Ser. I .... .
\end{abstract}

\section{Résumé}

Un nouveau schéma de volumes finis colocalisés pour les équations de Navier-Stokes incompressibles sur des maillages généraux non conformes. Un nouveau schéma volumes finis permet l'approximation des équations de Navier-Stokes sur des grilles générales. Ce schéma est basé sur une approximation de la formulation faible, et sur la définition d'opérateurs de gradient et divergence discrets consistants. On donne les grandes lignes de la preuve de convergence, ainsi que des résultats numériques obtenus pour un maillage non conforme. Un résultat auxiliaire est l'obtention d'un schéma de volumes finis convergent pour des problèmes de diffusion sur des grilles générales. Pour citer cet article : R. Eymard, R. Herbin, C. R. Acad. Sci. Paris, ...

Let $d=2$ or $d=3$, and $\Omega \subset \mathbb{R}^{d}$ be a polygonal connected domain. We focus here on the approximation of the pair $(\mathbf{u}, p)$ where $\mathbf{u}=\left(u^{(1)}, u^{(2)}\right)$ if $d=2, \mathbf{u}=\left(u^{(1)}, u^{(2)}, u^{(3)}\right)$ if $d=3$, stands for the velocity and $p$ for the pressure, solution of the incompressible steady Navier-Stokes equations

$$
\begin{aligned}
& -\nu \Delta \mathbf{u}+\nabla p+(\mathbf{u} \cdot \nabla) \mathbf{u}=f \text { in } \Omega \\
& \operatorname{div} \mathbf{u}=0 \text { in } \Omega
\end{aligned}
$$

Email addresses: Robert.Eymard@univ-mlv.fr (Robert Eymard), Raphaele.Herbin@latp.univ-mrs.fr (Raphaèle Herbin). 
with the usual notation $\mathbf{u} \cdot \nabla \mathbf{u}=\sum_{i=1}^{d} u^{(i)} \partial_{i} \mathbf{u}$, together with a homogeneous Dirichlet boundary condition for $\mathbf{u}$. A classical weak formulation of this problem is the following:

$$
\left\{\begin{array}{l}
\mathbf{u} \in H_{0}^{1}(\Omega)^{d}, p \in L^{2}(\Omega) \text { with } \int_{\Omega} p(\mathbf{x}) \mathrm{d} \mathbf{x}=0 \\
\nu \int_{\Omega} \nabla \mathbf{u}: \nabla \mathbf{v} \mathrm{d} \mathbf{x}-\int_{\Omega} p \operatorname{div} \mathbf{v} \mathbf{x} \mathbf{x}+b(\mathbf{u}, \mathbf{u}, \mathbf{v})=\int_{\Omega} \mathbf{f} \cdot \mathbf{v} \mathrm{d} \mathbf{x} \quad \forall \mathbf{v} \in H_{0}^{1}(\Omega)^{d}, \\
\operatorname{div} \mathbf{u}(\mathbf{x})=0 \text { for a.e. } \mathbf{x} \in \Omega,
\end{array}\right.
$$

where $\nu \in] 0,+\infty\left[\right.$ and $\mathbf{f} \in L^{2}(\Omega)^{d}, \nabla \mathbf{u}(\mathbf{x}): \nabla \mathbf{v}(\mathbf{x})=\sum_{i=1}^{d} \nabla u^{(i)} \cdot \nabla v^{(i)}$, and $b$ is the trilinear form over $H_{0}^{1}(\Omega)^{d}$ defined by: $b(\mathbf{u}, \mathbf{v}, \mathbf{w})=\sum_{k=1}^{d} \sum_{i=1}^{d} \int_{\Omega} u^{(i)}(\mathbf{x}) \partial_{i} v^{(k)}(\mathbf{x}) w^{(k)}(\mathbf{x}) \mathrm{d} \mathbf{x}$.

In [3], we proposed a colocated finite volume scheme on grids satisfying a so-called orthogonality condition, such as for instance triangular, rectangular and Voronoï meshes, allowing for a consistent approximation of diffusion fluxes by two-point fluxes. Colocated schemes are used in commercial codes (Fluent, CFX) or industrial codes [1] because they are easier to implement and allow for more general meshes than staggered schemes, even though the latter are known to be inherently stable. We extend here the study of [3] to general finite volume discretizations which do not have to respect the orthogonality condition and which may be non matching.

\section{Finite volume mesh and scheme}

We denote by $\mathcal{D}=(\mathcal{M}, \mathcal{E}, \mathcal{P})$ a finite volume mesh, where:

(i) $\mathcal{M}$ is a finite family of non empty connected open disjoint subsets of $\Omega$ (the "control volumes") such that $\bar{\Omega}=\cup_{K \in \mathcal{M}} \bar{K}$. For any $K \in \mathcal{M}$, let $\partial K=\bar{K} \backslash K, \mathrm{~m}_{K}>0$ and $h_{K}$ respectively denote the boundary, the measure and the diameter of $K$.

(ii) $\mathcal{E}$ is a finite family of disjoint subsets of $\bar{\Omega}$ (the "edges" of the mesh), such that, for all $\sigma \in \mathcal{E}, \sigma$ is a non empty closed subset of a hyperplane of $\mathbb{R}^{d}$, whose (d-1)-dimensional measure $\mathrm{m}_{\sigma}$ is strictly positive. We assume that, for all $K \in \mathcal{M}$, there exists a subset $\mathcal{E}_{K}$ of $\mathcal{E}$ such that $\partial K=\cup_{\sigma \in \mathcal{E}_{K}} \sigma$. We then denote by $\mathcal{M}_{\sigma}=\left\{K \in \mathcal{M}, \sigma \in \mathcal{E}_{K}\right\}$. We then assume that $\mathcal{E}$ is partitionned into $\mathcal{E}=\mathcal{E}_{\text {int }} \cup \mathcal{E}_{\text {ext }}$, such that, for all $\sigma \in \mathcal{E}_{\text {ext }}$ (boundary edge), $\mathcal{M}_{\sigma}$ has exactly one element and $\sigma \subset \partial \Omega$, and for all $\sigma \in \mathcal{E}_{\text {int }}$ (interior edge), $\mathcal{M}_{\sigma}$ has exactly two elements. For all $\sigma \in \mathcal{E}$, we denote by $\mathbf{x}_{\sigma}$ the barycenter of $\sigma$. For all $K \in \mathcal{M}$ and $\sigma \in \mathcal{E}_{K}$, we denote by $\mathbf{n}_{K, \sigma}$ the unit vector normal to $\sigma$ outward to $K$.

(iii) $\mathcal{P}$ is a family of points of $\Omega$ indexed by $\mathcal{M}$, denoted by $\mathcal{P}=\left(\mathbf{x}_{K}\right)_{K \in \mathcal{M}}$, such that for all $K \in \mathcal{M}$, $K$ is assumed to be $\mathbf{x}_{K}$-star-shaped, i.e. for all $x \in K$, the property $\left[\mathbf{x}_{K}, x\right] \subset K$ holds. Denoting by $d_{K, \sigma}$ the Euclidean distance between $\mathbf{x}_{K}$ and the hyperplane including $\sigma$, one assumes that $d_{K, \sigma}>0$.

The above definition applies to a large variety of meshes. Non matching meshes such as the one depicted in the final figure may be used, so that in particular, the common boundary of two neighbouring control volumes can include more than one edge. Note also that no hypothesis is made on the convexity of the control volumes, which enables that "hexahedra" with non planar faces can be used (such sets may have up to 12 faces if each non planar face consists in two triangles).

We introduce the set $H_{\mathcal{D}}(\Omega)$ of functions which are constant in each $K \in \mathcal{M}$, and for all regular function $\psi: \Omega \rightarrow \mathbb{R}\left(\right.$ resp. $\left.\Psi: \Omega \rightarrow \mathbb{R}^{d}\right)$, we denote by $P_{\mathcal{D}} \psi\left(\right.$ resp. $\left.\mathbf{P}_{\mathcal{D}} \Psi\right)$ the element of $H_{\mathcal{D}}(\Omega)\left(\operatorname{resp} . H_{\mathcal{D}}(\Omega)^{d}\right)$ such that $\left(P_{\mathcal{D}} \psi\right)_{K}=\psi\left(\mathbf{x}_{K}\right)$ (resp. $\left(\mathbf{P}_{\mathcal{D}} \Psi\right)_{K}=\Psi\left(\mathbf{x}_{K}\right)$ ) for all $K \in \mathcal{M}$. Let us write an approximation scheme for (1) in the following way: find $\left(\mathbf{u}_{\mathcal{D}}, p_{\mathcal{D}}\right) \in H_{\mathcal{D}}(\Omega)^{d} \times H_{\mathcal{D}}(\Omega)$ such that 


$$
\left\{\begin{array}{l}
\nu\left\langle\mathbf{u}_{\mathcal{D}}, \mathbf{v}\right\rangle_{\mathcal{D}}-\int_{\Omega} p_{\mathcal{D}} \operatorname{div}_{\mathcal{D}}(\mathbf{v}) \mathrm{d} \mathbf{x}+b_{\mathcal{D}}\left(\mathbf{u}_{\mathcal{D}}, \mathbf{u}_{\mathcal{D}}, \mathbf{v}, p_{\mathcal{D}}\right)=\int_{\Omega} \mathbf{f} \cdot \mathbf{v} \mathrm{d} \mathbf{x}, \forall \mathbf{v} \in H_{\mathcal{D}}(\Omega)^{d}, \\
\int_{\Omega} \operatorname{div}_{\mathcal{D}}\left(\mathbf{u}_{\mathcal{D}}\right) q \mathrm{~d} \mathbf{x}+S\left(p_{\mathcal{D}}, q\right)=0, \forall q \in H_{\mathcal{D}}(\Omega) \text { and } \int_{\Omega} p_{\mathcal{D}} \mathrm{d} \mathbf{x}=0 .
\end{array}\right.
$$

Let us first give the expression of $\langle\cdot, \cdot\rangle_{\mathcal{D}}$. For any edge $\sigma \in \mathcal{E}$ of the mesh, we define a linear mapping $U_{\sigma}$ on $H_{\mathcal{D}}(\Omega)$, such that for all regular function $\psi, U_{\sigma}\left(P_{\mathcal{D}} \psi\right)$ is an order 2 consistent approximation of $\psi\left(\mathbf{x}_{\sigma}\right)$. Since we consider Dirichlet boundary conditions, we set $U_{\sigma}(u)=0$ for all $u \in H_{\mathcal{D}}(\Omega)$ and $\sigma \in \mathcal{E}_{\text {ext }}$. This allows to define a consistent approximate gradient of the elements of $H_{\mathcal{D}}(\Omega)$ by

$$
\left(\nabla_{\mathcal{D}} u\right)_{K}=\frac{1}{\mathrm{~m}_{K}} \sum_{\sigma \in \mathcal{E}_{K}} \mathrm{~m}_{\sigma}\left(U_{\sigma}(u)-u_{K}\right) \mathbf{n}_{K, \sigma}\left(=\frac{1}{\mathrm{~m}_{K}} \sum_{\sigma \in \mathcal{E}_{K}} \mathrm{~m}_{\sigma} U_{\sigma}(u) \mathbf{n}_{K, \sigma}\right) \forall u \in H_{\mathcal{D}}(\Omega),
$$

We then define the linear mappings $\left(R_{K, \sigma}\right)_{K \in \mathcal{M}, \sigma \in \mathcal{E}_{K}}$ on $H_{\mathcal{D}}(\Omega)$ by

$$
R_{K, \sigma} u=U_{\sigma}(u)-u_{K}-\left(\nabla_{\mathcal{D}} u\right)_{K} \cdot\left(\mathbf{x}_{\sigma}-\mathbf{x}_{K}\right), \forall u \in H_{\mathcal{D}}(\Omega), \forall K \in \mathcal{M}, \forall \sigma \in \mathcal{E}_{K},
$$

For a given family $\left(\alpha_{K}\right)_{K \in \mathcal{M}}$ of positive real numbers, let us define the following inner product on $H_{\mathcal{D}}(\Omega)$ :

$$
\langle u, v\rangle_{\mathcal{D}}:=\int_{\Omega} \nabla_{\mathcal{D}} u(\mathbf{x}) \cdot \nabla_{\mathcal{D}} v(\mathbf{x}) \mathrm{d} \mathbf{x}+\sum_{K \in \mathcal{M}} \alpha_{K} \sum_{\sigma \in \mathcal{E}_{K}} \frac{\mathrm{m}_{\sigma}}{d_{K, \sigma}} R_{K, \sigma} u R_{K, \sigma} v, \forall u, v \in H_{\mathcal{D}}(\Omega) .
$$

Note that the bilinear form defined by $\int_{\Omega} \nabla_{\mathcal{D}} u(\mathbf{x}) \cdot \nabla_{\mathcal{D}} v(\mathbf{x}) \mathrm{d} \mathbf{x}$ does not define an inner product; the bilinear form $\langle\cdot, \cdot\rangle_{\mathcal{D}}$ is definite thanks to the parameters $\alpha_{K}>0$. For $\mathbf{u}, \mathbf{v} \in H_{\mathcal{D}}(\Omega)^{d}$, we set $\langle\mathbf{u}, \mathbf{v}\rangle_{\mathcal{D}}=$ $\sum_{i=1}^{d}\left\langle u^{(i)}, v^{(i)}\right\rangle_{\mathcal{D}}$, and $\mathbf{U}_{\sigma}(\mathbf{u})=\left(U_{\sigma}\left(u^{(1)}\right), \ldots, U_{\sigma}\left(u^{(d)}\right)\right)$. We can now define $\operatorname{div} \mathcal{D} \mathbf{v}$ by

$$
\left(\operatorname{div}_{\mathcal{D}} \mathbf{v}\right)_{K}=\frac{1}{\mathrm{~m}_{K}} \sum_{\sigma \in \mathcal{E}_{K}} \mathrm{~m}_{\sigma} \mathbf{U}_{\sigma}(\mathbf{v}) \cdot \mathbf{n}_{K, \sigma}=\sum_{i=1}^{d}\left(\nabla_{\mathcal{D}} v^{(i)}\right)^{(i)}, \forall \mathbf{v} \in H_{\mathcal{D}}(\Omega)^{d} .
$$

Because of the colocated nature of the scheme, the approximation of the mass conservation (second equation of (2)) includes a stabilization term $S(\cdot, \cdot)$, which is defined in the following way. Let $\left(\lambda_{\sigma}\right)_{\sigma \in \mathcal{E}_{\text {int }}}$ be a given family of positive real numbers; we define:

$$
S(p, q)=\sum_{\sigma, \mathcal{M}_{\sigma}=\{K, L\}} \lambda_{\sigma} \mathrm{m}_{\sigma}\left(h_{K}+h_{L}\right)\left(p_{K}-p_{L}\right)\left(q_{K}-q_{L}\right), \forall p, q \in H_{\mathcal{D}}(\Omega)
$$

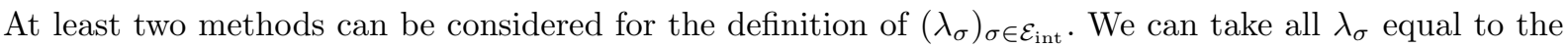
same value; this yields a stabilization term inspired by [2]. Alternately, we can define a partition of $\mathcal{M}$, denoted $\mathcal{C}$, and set $\lambda_{\sigma}=\lambda$ if there exists $G \in \mathcal{C}$ (such $G \subset \mathcal{M}$ is called "a cluster") with $\mathcal{M}_{\sigma} \subset G$, otherwise we set $\lambda_{\sigma}=0$ (see [4]). Both methods show good results. For $\sigma \in \mathcal{E}_{\text {int }}$, with $\mathcal{M}_{\sigma}=\{K, L\}$, defining $\Phi_{K, \sigma}(\mathbf{u}, p)=\mathrm{m}_{\sigma}\left(\mathbf{U}_{\sigma}(\mathbf{u}) \cdot \mathbf{n}_{K, \sigma}+\lambda_{\sigma}\left(h_{K}+h_{L}\right)\left(p_{K}-p_{L}\right)\right)$, the second equation of (2) also reads: $\sum_{\sigma \in \mathcal{E}_{K} \cap \mathcal{E}_{\text {int }}} \Phi_{K, \sigma}(\mathbf{u}, p)=0$. Finally, we define the centred approximation $b_{\mathcal{D}}\left(\mathbf{u}_{\mathcal{D}}, \mathbf{u}_{\mathcal{D}}, \mathbf{v}, p_{\mathcal{D}}\right)$ of the trilinear form $b$ by

$$
b_{\mathcal{D}}(\mathbf{u}, \mathbf{v}, \mathbf{w}, p)=\sum_{K \in \mathcal{M}} \mathbf{w}_{K} \cdot\left(\sum_{\sigma \in \mathcal{E}_{K}, \mathcal{M}_{\sigma}=\{K, L\}} \Phi_{K, \sigma}(\mathbf{v}, p) \frac{\mathbf{u}_{K}+\mathbf{u}_{L}}{2}\right),
$$

which allows for the property $b_{\mathcal{D}}\left(\mathbf{u}_{\mathcal{D}}, \mathbf{u}_{\mathcal{D}}, \mathbf{u}_{\mathcal{D}}, p_{\mathcal{D}}\right)=0$. Note that this approximation depends on the pressure through the stabilisation term in the discrete mass flux $\Phi_{K, \sigma}\left(\mathbf{u}_{\mathcal{D}}, p_{\mathcal{D}}\right)$. An upstream weighted approximation, satisfying the same property, could as well be defined. 


\section{Convergence analysis}

As in [3], the convergence of the scheme is proven, in the case of constant value for all $\lambda_{\sigma}$, thanks to the following arguments:

(i) Setting $\mathbf{v}=\mathbf{u}_{\mathcal{D}}$ and $q=p_{\mathcal{D}}$ in (2), we can get, thanks to the property $b_{\mathcal{D}}\left(\mathbf{u}_{\mathcal{D}}, \mathbf{u}_{\mathcal{D}}, \mathbf{u}_{\mathcal{D}}, p_{\mathcal{D}}\right)=0$, some bounds on $\left\langle\mathbf{u}_{\mathcal{D}}, \mathbf{u}_{\mathcal{D}}\right\rangle_{\mathcal{D}}$ and $S\left(p_{\mathcal{D}}, p_{\mathcal{D}}\right)$. These bounds yield the existence of a solution to the scheme thanks to a topological degree argument, and allow to apply a compactness result on the set of approximate velocities.

(ii) We derive a bound on $\left\|p_{\mathcal{D}}\right\|_{L^{2}(\Omega)}$, following Nečas results, hence proving the weak convergence in $L^{2}(\Omega)$ of the approximate pressure.

(iii) Let $\left(\mathcal{D}_{n}\right)_{n \in \mathbb{N}}$ be a sequence of meshes with mesh size tending to 0 as $n$ tends to $+\infty$, let $\left(\mathbf{w}_{n}\right)_{n \in \mathbb{N}} \subset$ $\left(H_{\mathcal{D}_{n}}(\Omega)\right)^{d}$ be a sequence of functions such that the term $\left\langle\mathbf{w}_{n}, \mathbf{w}_{n}\right\rangle_{\mathcal{D}_{n}}$ remains bounded, and which converges in $L^{2}(\Omega)$ to some function $\mathbf{w} \in H_{0}^{1}(\Omega)$, as $n$ tends to $+\infty$; it may then be shown that, for any regular function $\Psi: \Omega \rightarrow \mathbb{R}^{d}$ vanishing at the boundary, the term $\left\langle\mathbf{w}_{n}, \mathbf{P}_{\mathcal{D}_{n}} \Psi\right\rangle_{\mathcal{D}_{n}}$ tends to $\int_{\Omega} \nabla \mathbf{w}: \nabla \Psi \mathrm{d} \mathbf{x}$, as $n$ tends to $+\infty$. The proof uses the order 2 consistency property of $\mathbf{U}_{\sigma}\left(\mathbf{P}_{\mathcal{D}_{n}} \Psi\right)$ with $\Psi\left(\mathbf{x}_{\sigma}\right)$. During the course of this proof, we get that the approximate solution for the following diffusion problem: find $u \in H_{\mathcal{D}}(\Omega)$ such that $\left\langle u^{(\mathcal{D})}, v\right\rangle_{\mathcal{D}}=\int_{\Omega} f(\mathbf{x}) v(\mathbf{x}) \mathrm{d} \mathbf{x}$, for all $v \in H_{\mathcal{D}}(\Omega)$, converges to the weak solution $u \in H_{0}^{1}(\Omega)$ of $-\Delta u=f$ with $u=0$ on $\partial \Omega$.

(iv) We finally get the convergence of any approximate finite volume solution (that is a solution of (2), and up to a subsequence) to a weak solution of (1), by passing to the limit, taking, for any regular function $\Psi, \mathbf{P}_{\mathcal{D}_{n}} \Psi$ as a test function in (2), and concluding by density. This step again uses the order 2 consistency property of functions $\mathbf{U}_{\sigma}$ and the bound on $S\left(p_{\mathcal{D}}, p_{\mathcal{D}}\right)$.

\section{Numerical results}

Let us conclude this paper with some numerical results: we consider the classical lid driven cavity, with $R e=1000$, taking $\alpha_{K}=1$ for all $K \in \mathcal{M}$. The following figure presents on the left the grid used for the computation of the approximate solution, which shows that there is no need that the mesh be conforming in the usual finite element sense, nor suited for two-points fluxes; the right side of the figure shows the streamlines obtained on this grid.

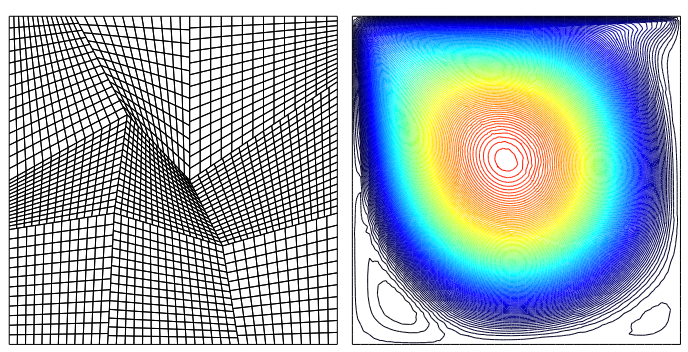

\section{References}

[1] F. Archambeau, N. Mehitoua, and M. Sakiz. Code saturne: A finite volume code for turbulent flows. Int. J. Finite Volumes, 1(1), 2004. http://www.latp.univ-mrs.fr/IJFV.

[2] F. Brezzi and J. Pitkäranta. On the stabilization of finite element approximations of the Stokes equations. In Efficient solutions of elliptic systems (Kiel, 1984), volume 10 of Notes Numer. Fluid Mech., pages 11-19. Vieweg, Braunschweig, 1984 .

[3] R. Eymard, R. Herbin, and J.C. Latché. Convergence analysis of a colocated finite volume scheme for the incompressible navier-stokes equations on general 2 or 3d meshes. SIAM J. Numer. Anal., 45(1):1-36, 2007.

[4] R. Eymard, R. Herbin, J.C. Latché, and B. Piar. On colocated finite volume scheme for the incompressible flow problems. submitted, http://hal.archives-ouvertes.fr/, 2006. 